\title{
BIBLIOGRAPHY
}

1. A. Borel, Groupes linéaires algébriques, Ann. of Math. vol. 64(1956) pp. 20-80.

2. C. Hermite, Oeuvres complètes, Vol. 1, Paris, Gauthier-Villars, 1905.

3. Y. Matsushima, Espaces homogènes de Stein des groupes de Lie complexes, Nagoya Math. J. vol. 16 (1960) pp. 205-218.

4. G. D. Mostow, Self-adjoint group, Ann. of Math. vol. 62 (1955), pp. 44-55.

5. T. Ono, Sur une propriêté arithmétique des groupes algébriques commutatifs, Bull. Soc. Math. France vol. 85 (1957) pp. 307-323.

6. K. G. Ramanathan, Unit of fixed points in involutorial algebras, Proceedings of the International Symposium on Algebraic Number Theory, Tokyo, 1955.

7. Séminaire S. Lie, Théorie des algèbres de Lie, Topologie des groupes de Lie, Paris, 1954-1955.

8. C. L. Siegel, Einheiten quadratischer Formen, Abh. Math. Sem. Univ. Hamburg vol. 13 (1939) pp. 209-239.

9. A. Weil, Discontinuous subgroups of classical groups, Notes, University of Chicago, 1958.

The Institute for Advanced Study AND

Columbia University

\section{SOME PROPERTIES OF ADELE GROUPS ATTACHED TO ALGEBRAIC GROUPS}

BY ARMAND BOREL

Communicated by Deane Montgomery, July 22, 1961

This note is a sequel to the previous one [1], and is devoted to some applications of the results of the latter to adele groups. The results are valid for linear algebraic groups defined over number fields, but this case is easily reduced to that of groups defined over $\mathbf{Q}[\mathbf{3}$, Chapter I], to which we shall limit ourselves for simplicity.

The notation of [1] is freely used. For the unexplained notions concerning adeles, see $[2 ; 3]$.

1. Adeles. Let $G$ be a connected algebraic linear group defined over Q. The adele group attached to $G$ is denoted by $G_{A}$. The group $G_{\mathcal{B}}$ is identified with the subgroup of principal adeles of $G_{A}$; it is discrete. We put

$$
G_{A}^{0}=G_{\mathrm{R}} \times \prod_{p \text { prime }} G_{Z_{\mathrm{p}}} \quad\left(\boldsymbol{Z}_{p} \text { : ring of } p \text {-adic integers }\right) .
$$

By definition, $G_{A}^{0}$, endowed with the product topology, is an open subgroup of $G_{A}$. The group $G$ is said to be of type $(F)$ if $G_{A}$ is the union of a finite number of double cosets $G_{A}^{0} \cdot x \cdot G_{Q}\left(x \in G_{A}\right)$ [2]. 
Let $X_{Q}(G)$ be the group of rational characters of $G$, defined over $Q$. Each $\chi \in X_{Q}(G)$ induces a continuous homomorphism $\chi_{A}: G_{A} \rightarrow I(Q)$, where $I(Q)$ is the idele group of $Q$. Composed with the norm mapping $\eta: I(Q) \rightarrow R^{+}$, it yields a continuous homomorphism $\eta \circ \chi_{A}: G_{A}$ $\rightarrow R^{+}$. Let ${ }_{N} G_{A}$ be the intersection of the kernels of the homomorphisms $\eta \circ \chi_{A}\left(\chi \in X_{Q}(G)\right)$. It is unimodular [2, Proposition 11] and contains $G_{Q}$. The group $G$ is said to be of type $(M)$ (resp. $(C)$ ), if ${ }_{N} G_{A} / G_{Q}$ has finite invariant measure (resp. is compact), [2].

TheOREM 1. Let $G$ be a connected algebraic group, defined over $\mathbf{Q}$. Then $G$ is of type $(F)$ and of type $(M)$. It is of type $(C)$ if and only if every unipotent element of $G_{Q}$ belongs to the radical of $G_{Q}$. The quotient $G_{A} / G_{Q}$ is compact if and only if $G$ is of type $(C)$ and $X_{Q}(G)=1$.

In view of the results of [2], it is enough to prove this for reductive groups. To this end, a fundamental set for $G_{Q}$ in $G_{A}$ is constructed by a method which is quite analogous to the one outlined in $[1, \S 3]$. This yields condition $(F)$, and reduces the other assertions to Theorems 1,3 of [1].

2. Let $G L(m, A)$ and $S L(m, A)$ be the adele-groups attached to $G L(m, \boldsymbol{Q})$ and $S L(m, \boldsymbol{Q})$ respectively. These are groups of automorphisms of $A^{m}$, the free module of rank $m$ over the adele ring $A$ of $\boldsymbol{Q}$. A rational homomorphism $\pi: G \rightarrow G L(m, \boldsymbol{C})$, defined over $\boldsymbol{Q}$, induces a continuous homomorphism $\pi_{A}: G_{A} \rightarrow G L(m, A)$, for which the following analogue to Theorem 2 in [1] holds:

THEOREM 2. Let $G$ be a connected reductive algebraic group, defined over $\boldsymbol{Q}, \pi: G \rightarrow G L(m, C)$ a rational representation, defined over $Q$, and $v \in Q^{m}$ a point whose orbit is closed. Then $v \cdot \pi_{A}\left(G_{A}\right) \cap Q^{m}$ is the union of a finite number of orbits of $G_{Q}$.

(In this statement $\mathbf{Q}^{m}$ is identified with the principal adeles of $A^{m}$.)

3. Principal homogeneous spaces. Let $G$ be a connected algebraic group defined over $\boldsymbol{Q}$. A principal homogeneous space $V$ of $G$, over $\boldsymbol{Q}$, is a variety defined over $\boldsymbol{Q}$, on which $G$ acts as an algebraic transformation group by means of a morphism $G \times V \rightarrow V$ defined over $\boldsymbol{Q}$, and such that for any $v \in V$, the map $g \rightarrow g \cdot v$ is a birational biregular map of $G$ onto $V$. (Since we are in characteristic zero, this last condition is equivalent to: $G$ is simply transitive on $V$.) Two such spaces $V, V^{\prime}$ are isomorphic over $Q$ if there exists a $G$-equivariant birational biregular map of $V$ onto $V^{\prime}$, defined over $\mathbf{Q}$. 
THEOREM 3. Let $G$ be a connected reductive group defined over $\mathbf{Q}$. Then the principal homogeneous spaces over $\mathbb{Q}$ which have rational points in all completions of $\boldsymbol{Q}$ form a finite number of isomorphism classes.

Assume $G \subset S L(n, C)$. Let $\pi: S L(n, C) \rightarrow G L(m, C)$ be a rational representation defined over $\boldsymbol{Q}$, for which there exists a point $v \in{Q^{m}}^{m}$ whose orbit $V$ is closed and whose isotropy group is $G$ [1, Lemma 2]. It is not difficult to show that the orbits of $S L(n, \boldsymbol{Q})$ in $v \cdot \pi_{A}(S L(n, A))$ are in 1-1 correspondence with the isomorphism classes of principal homogeneous spaces over $\boldsymbol{Q}$ which have rational points in all completions of $\boldsymbol{Q}$, hence Theorem 3 follows from Theorem 2 .

REMARKs. (1) Theorem 1 was known for solvable groups [2] and for many classical groups [3].

(2) Theorem 3 is well known for algebraic tori. In this case, the principal homogeneous spaces in question may form several classes. It is not known to the author whether there may be more than one class when $G$ is semi-simple (and connected). For further remarks on this question, see S. Lang, Bull. Amer. Math. Soc. vol. 66 (1960) pp. 240-249.

\section{BIBLIOGRAPHY}

1. A. Borel and Harish-Chandra, Arithmetic subgroups of algebraic groups, Bull. Amer. Math. Soc. vol. 67 (1961) pp. 579-583.

2. T. Ono, On some arithmetic properties of linear algebraic groups, Ann. of Math. vol. 70 (1959) pp. 266-290.

3. A. Weil, Adeles and algebraic groups, Notes, The Institute for Advanced Study, Princeton, 1961.

The Institute for Advanced Study 